\title{
MicroRNA-126 inhibits the migration and invasion of endometrial cancer cells by targeting insulin receptor substrate 1
}

\author{
XIUMIN ZHAO, DANYANG ZHU, CAILING LU, DEWEN YAN, LIFENG LI and ZHOUFANG CHEN \\ Department of Obstetrics and Gynecology, The First People's Hospital of Taizhou City, Taizhou, Zhejiang 318020, P.R. China
}

Received January 22, 2015; Accepted November 19, 2015

DOI: $10.3892 / 01.2015 .4001$

\begin{abstract}
MicroRNAs (miRs) have been demonstrated to serve important roles in the development and progression of human cancer, primarily through the direct targeting of oncogenes or tumor suppressors. It has been previously suggested that miR-126 may be associated with endometrial cancer (EC). However, the exact role of miR-126 in the migration and invasion of EC cells has not yet been studied. The present study demonstrated that the expression of miR-126 was significantly decreased in EC tissues when compared with matched normal adjacent tissues. The current study reverse transcriptionquantitative polymerase chain reaction was performed in order to examine the expression level of miR-126. Wound healing and transwell assays were used to examine cell migration and invasion. A luciferase reporter assay was used to determine the targeting relationship and western blotting assay was performed to detect the protein expression. Furthermore, the overexpression of miR-126 significantly inhibited EC SKOV3 cell migration and invasion. Molecular mechanism investigation established that insulin receptor substrate 1 (IRS1) functioned as a direct miR-126 target, and its expression was negatively regulated by miR-126 at a post-transcriptional level in the SKOV3 cells. Additionally, the overexpression of IRS1 reversed the inhibitory effect of miR-126 overexpression on SKOV3 cell migration and invasion. In conclusion, the current study demonstrated that miR-126 inhibited EC cell migration and invasion, at least partially through the direct targeting of IRS1, suggesting that miR-126 may aid the treatment of EC metastasis.
\end{abstract}

\section{Introduction}

Endometrial cancer (EC) is one of the most malignant tumors to threaten the health of women (1). Over the past

Correspondence to: Professor Danyang Zhu, Department of Obstetrics and Gynecology, The First People's Hospital of Taizhou City, 218 Hengjie Road, Taizhou, Zhejiang 318020, P.R. China

E-mail: doctorzhudanyang@163.com

Key words: endometrial cancer, microRNA-126, insulin receptor substrate 1 , migration, invasion decades, unremitting efforts to improve the diagnostics and subsequent treatment of EC have been attempted. However, the incidence rate of EC has risen, and the average age of EC onset has fallen (2). The age-standardised incidence is 9.1 per 100,000 women per year in more developed countries, compared with 1.7 per 100,000 per year in less developed countries (3). The dysregulation of oncogenes and/or tumor suppressors has been demonstrated to serve a role in EC metastasis $(1,4)$. Therefore, the development of potential molecular targets indicates promise for the treatment of EC progression.

MicroRNAs (miRs) are short, non-coding RNAs, which may cause mRNA degradation or the inhibition of protein translation by directly binding to the 3'-untranslated region (UTR) of their target mRNAs (5). Through modulation of the protein expression of their target genes, which act as oncogenes or tumor suppressors, miRs are closely associated with the development and progression of human cancer, including EC (4). Among these miRs, miR-126 typically employs a suppressive role in numerous types of cancer, including non-small cell lung cancer, cervical cancer, gastric cancer, colon cancer, clear-cell renal cell carcinoma and chronic myelogenous leukemia (6-10). Recently, miR-126 was suggested to function as a tumor suppressor in EC (1). However, the exact role of miR-126 in the mediation of EC cell migration and invasion has not yet been studied.

Insulin receptor substrate 1 (IRS1) encodes a protein that may be phosphorylated by the insulin receptor tyrosine kinase, and mutations in IRS1 are associated with type II diabetes and susceptibility to insulin resistance $(11,12)$. The role of IRS1 in EC was identified in the study by Hua et al, which demonstrated that there is an excessive activation of IRS1 in EC (13). However, the regulatory mechanisms that underlie the expression of IRS1 in EC have yet to be studied.

The present study aimed to investigate the expression of miR-126 in EC tissues. Furthermore, the role of miR-126 in the regulation of the migratory and invasive capacities of EC cells was also investigated, as well as the underlying mechanisms involving IRS1.

\section{Materials and methods}

Tissue specimen collection. The current study was approved by the Ethical Committee of The First People's Hospital of Taizhou City (Taizhou, Zhejiang, China). A total of 11 EC tissues and matched adjacent normal tissues were obtained 


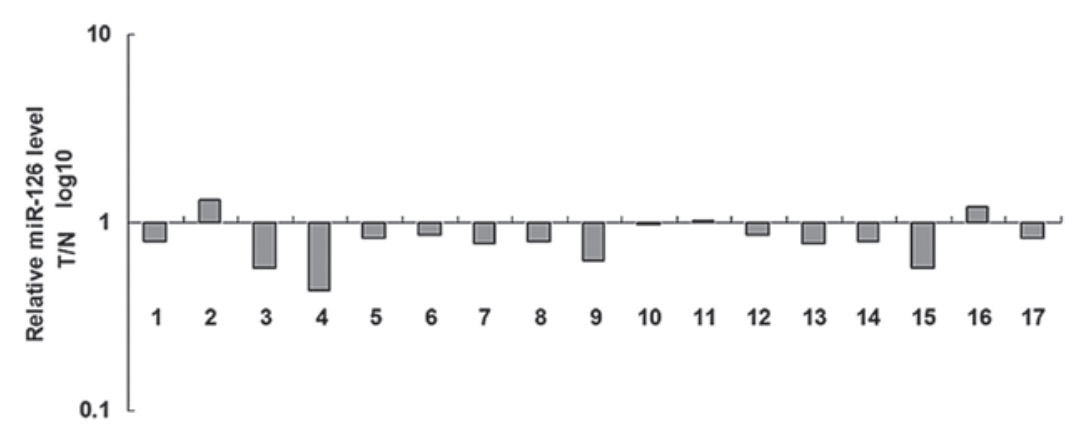

Figure 1. Reverse transcription-quantitative polymerase chain reaction was performed to examine the relative expression of miR-126 in the endometrial cancer tissues (tumor) and the matched normal adjacent tissues (normal). T/N, tumor/normal; miR, microRNA.

from the Department of Obstetrics and Gynecology, The First People's Hospital of Taizhou City during resection surgery. All participants provided full written consent prior to participation in the study. All tissues were immediately snap-frozen in liquid nitrogen following surgical removal and were stored at $-70^{\circ} \mathrm{C}$ until use.

Cell culture. The human RL95-2 cell line was obtained from the Cell Bank of the Chinese Academy of Sciences (Shanghai, China). The cells were cultured in Dulbecco's modified Eagle's medium with $10 \%$ fetal bovine serum, at $37^{\circ} \mathrm{C}$ in a humidified incubator containing $5 \% \mathrm{CO}_{2}$.

Reverse transcription-quantitative polymerase chain reaction $(R T-q P C R)$. Total RNA was extracted using the TRIzol ${ }^{\circledR}$ reagent (Thermo Fisher Scientific, Inc., Waltham, MA, USA). For the detection of miRs, a TaqMan ${ }^{\circledR}$ MicroRNA Reverse Transcription kit (Thermo Fisher Scientific, Inc.) was used to convert RNA into complementary DNA, according to the manufacturer's protocols. qPCR was then performed using $1000 \mathrm{ng}$ with the All-in-One miRNA qRT-PCR Detection kit (GeneCopoeia, Inc., Rockville, MD, USA) on the 7500 Real-Time PCR system (Applied Biosystems; Thermo Fisher Scientific, Inc.). The primer sequences: For miR-126 forward: TCGTACCGTGAGTAATAATGCG; for U6 forward: CTCGCTTCGGCAGCACA. For both reverse: Universal qPCR primer provided by the kit. The PCR conditions: $95^{\circ} \mathrm{C}$ for $5 \mathrm{~min}$, and 40 cycles of denaturation at $95^{\circ} \mathrm{C}$ for $15 \mathrm{sec}$ and annealing/elongation at $60^{\circ} \mathrm{C}$ for $30 \mathrm{sec}$. The U6 gene was used as an internal reference. The relative miR-126 expression was normalized to U6. The relative expression was analyzed by the $2^{-\Delta \Delta \mathrm{Cq}}$ method.

Western blot analysis. Tissues and cells were solubilized in cold radioimmunoprecipitation assay lysis buffer. Proteins were separated with $10 \%$ sodium dodecyl sulfate-polyacrylamide gel electrophoresis, and were transferred onto a polyvinylidene difluoride (PVDF) membrane. The PVDF membrane was incubated with phosphate-buffered saline, containing 5\% milk, overnight at $4^{\circ} \mathrm{C}$. Subsequently, the PVDF membrane was incubated with monoclonal mouse anti-human IRS1 and mouse anti-human glyceraldehyde 3-phosphate dehydrogenase (GAPDH) primary antibodies (respective cat nos. ab201644 and ab8245, respective dilutions, 1:500 and 1:200, Abcam, Cambridge, MA, USA) at room temperature for $3 \mathrm{~h}$, respectively.
This was followed by incubation with rabbit anti-mouse $\mathrm{IgG}$ secondary antibodies (cat no. ab97046, dilution 1:20,000, Abcam), also at room temperature for $1 \mathrm{~h}$. An enhanced chemiluminescence kit (Pierce Biotechnology, Inc., Rockford, IL, USA) was then used to detect chemiluminescence. The relative protein expression was analyzed by Image-Pro Plus software (version 6.0; Media Cybernetics, Inc., Rockville, MD, USA), represented as the density ratio versus GAPDH.

Transfection. Lipofectamine ${ }^{\circledR} 2000$ (Thermo Fisher Scientific, Inc.) was used to perform cell transfection, following the manufacturer's protocols. For functional analysis, the SKOV3 cells were transfected with scrambled miRNA as a negative control (NC), miR-126 mimic or miR-126 inhibitor (Thermo Fisher Scientific, Inc.), or were co-transfected with miR-126 mimics and IRS1 plasmid (all purchased from Nlunbio, Changsha, China), respectively.

Dual luciferase reporter assay. A QuikChange ${ }^{\circledR}$ II XL Site-Directed Mutagenesis kit (Agilent Technologies, Inc., Santa Clara, CA, USA) was used to generate a mutant-type 3'-UTR of IRS1, according to the manufacturer's protocols. The wild- or mutant-type 3'-UTRs of IRS1 were inserted into the psiCHECK2 vector (Promega Corporation, Madison, WI, USA), respectively. For the luciferase reporter assay, the SKOV3 cells were cultured to $\sim 60 \%$ confluence in a 24 -well plate, and were then transfected with psiCHECK2-IRS1-3'-UTR or psiCHECK2-mutant IRS1-3'-UTR vector, with or without $100 \mathrm{nM}$ miR-126 mimics, respectively. Following incubation for 48 h, a Dual-Luciferase ${ }^{\circledR}$ Reporter assay system (Promega Corporation) was used to determine luciferase activity on the LD400 Luminometer (Beckman Coulter, Inc., Fullerton, CA, USA). Renilla luciferase activity was normalized to firefly luciferase activity.

Statistical analysis. All data are presented as the mean \pm standard deviation. Differences were analyzed using a one-way analysis of variance. SPSS software, version 18.0 (SPSS, Inc., Chicago, IL, USA), was used to perform statistical analyses. $\mathrm{P}<0.05$ was considered to indicate a statistically significant difference.

\section{Results}

miR-126 is frequently downregulated in EC tissues. RT-qPCR was utilized to detect the expression of miR-126 in the EC tissues and matched normal adjacent tissues. As presented in 
A
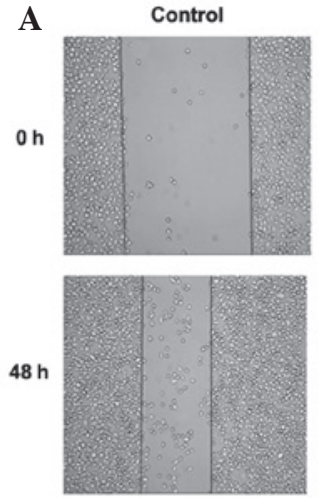

NC
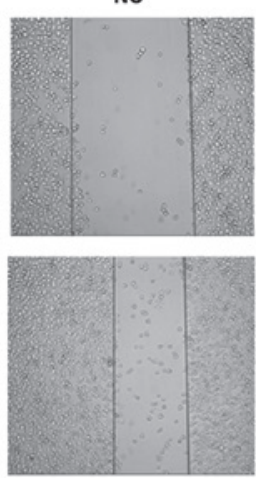

miR-126
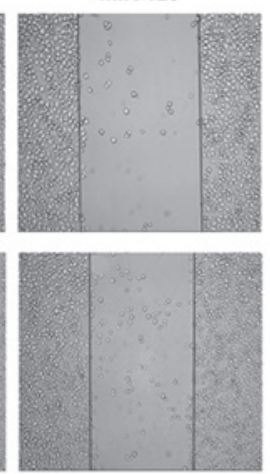

B
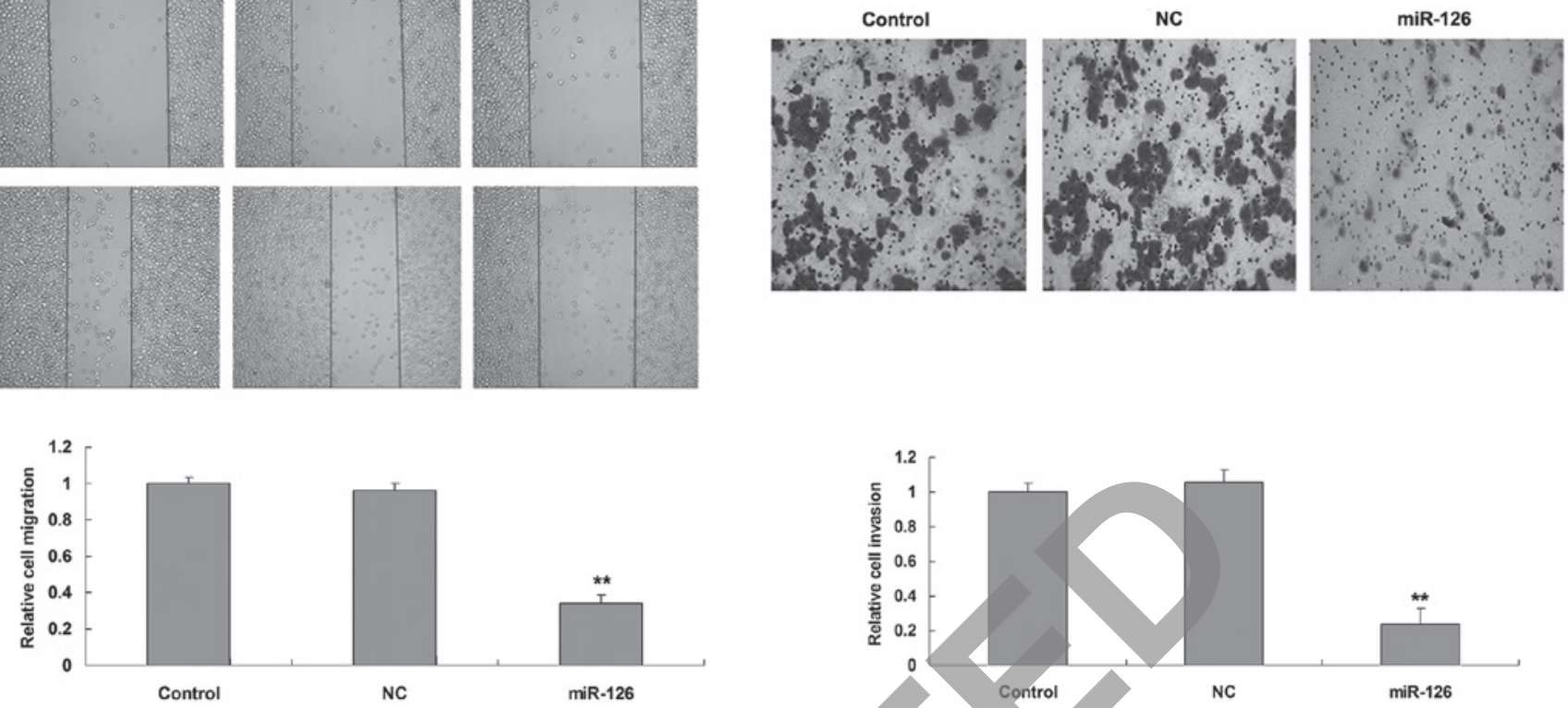

Figure 2. (A) A scratch assay was performed to determine the migratory capacity of the SKOV3 cells transfected with scramble miRNA as a NC or miR-126 mimics, respectively. (B) A Transwell assay was performed to determine the invasive capacity of the SKOV3 cells that had been transfected with scramble miRNA as a NC or miR-126 mimics, respectively. Magnification, x200. Control, SKOV3 cells without any transfection; NC, negative control; miR, microRNA. *P<0.01 vs. control.

A

B

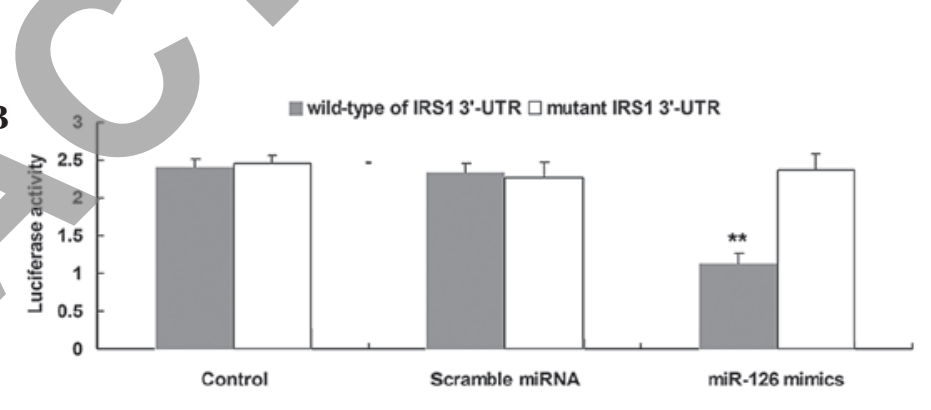

C
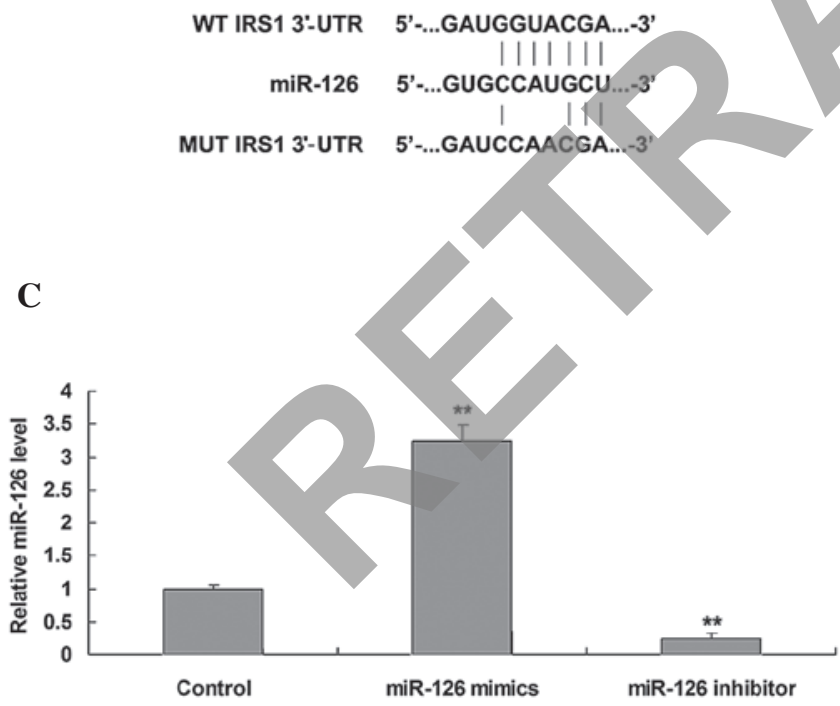

D

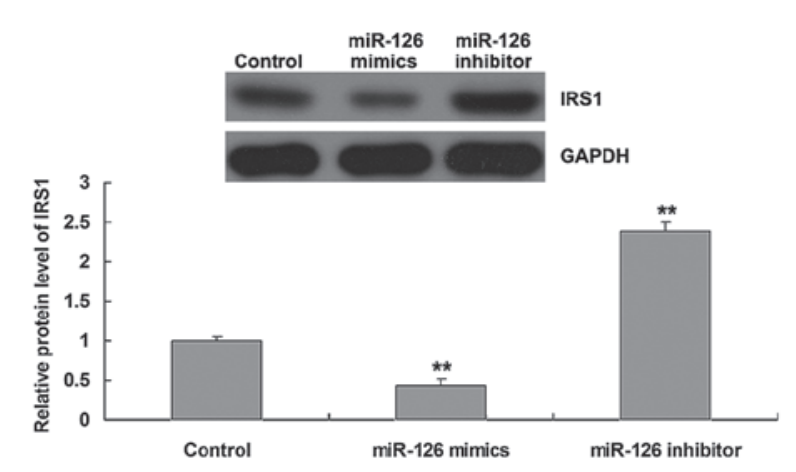

Figure 3. (A) Seed sequences of miR-126 in the WT or MUT 3'-UTR of IRS1 are indicated. (B) Luciferase reporter assay data identified that cotransfection of the SKOV3 cells with miR-126 and WT IRS1 3'-UTR produced a significant decrease in luciferase activity, whereas cotransfection with MUT IRS1 3'-UTR and miR-126 mimics demonstrated no difference with the control group. Control, cells cotransfected with blank vector and WT IRS1 3'-UTR, or MUT IRS1 3'-UTR. (C) Reverse transcription-quantitative polymerase chain reaction was performed to examine the expression of miR-126 in the SKOV3 cells transfected with miR-126 mimics or miR-126 inhibitor, respectively. Control, SKOV3 cells without any transfection. (D) Western blot analysis was performed to examine the protein level of IRS1 in the SKOV3 cells transfected with miR-126 mimics or miR-126 inhibitor, respectively. GAPDH was used as an internal reference. Control, SKOV3 cells without any transfection ${ }^{* *} \mathrm{P}<0.01$ vs. control. WT, wild-type; MUT, mutant-type; UTR, untranslated region; IRS1, insulin receptor substrate 1; miR, microRNA; GAPDH, glyceraldehyde 3-phosphate dehydrogenase.

Fig. 1, the expression of miR-126 was frequently reduced in the EC tissues compared with the matched adjacent normal tissues.

Upregulation of miR-126 suppresses EC cell migration and invasion. The role of miR-126 in mediating EC cell migration and invasion was investigated. Following transfection of the SKOV3 cells with miR-126 mimics or scramble miRNA mimics as a NC, the transfection efficiency was determined to be satisfactory. Subsequently, cell migration was analyzed by performing a scratch assay (Fig. 2A). As presented in 
A
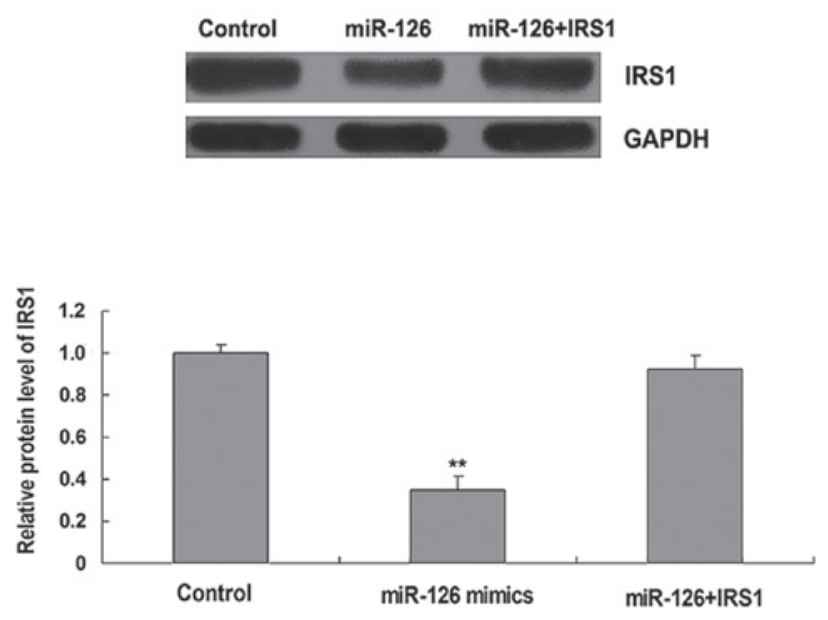

B
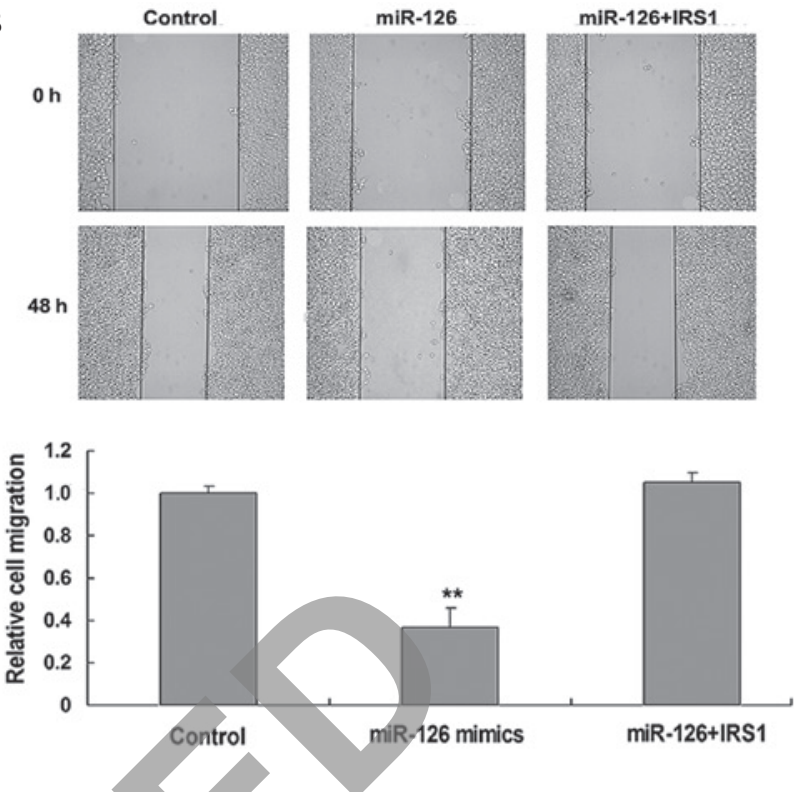

C
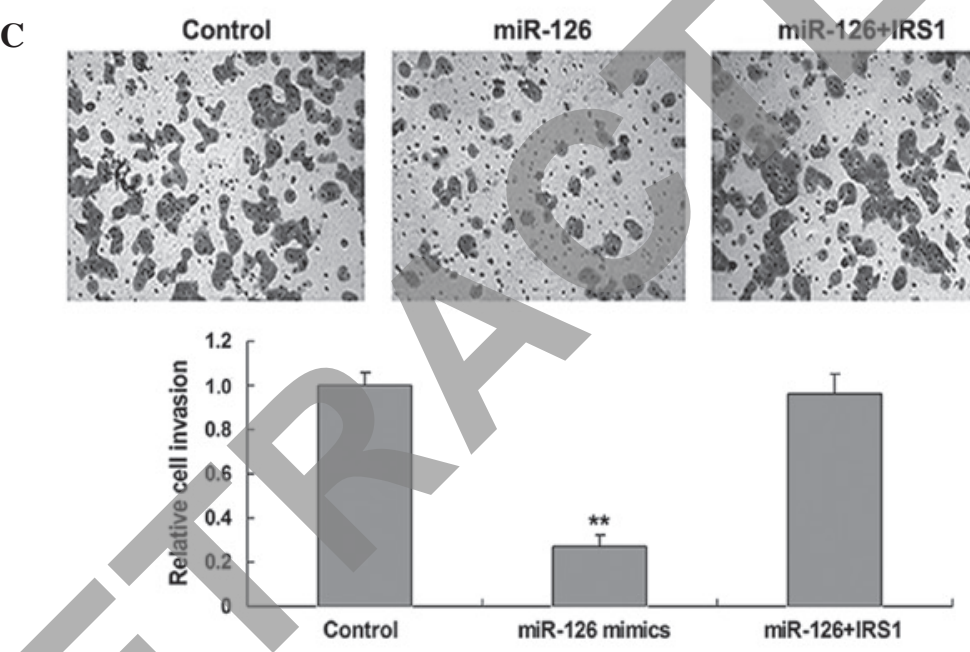

Figure 4. (A) Western blot analysis was performed to examine the protein level of IRS1 in the SKOV3 cells that had been transfected with miR-126 mimics, or cotransfected with miR-126 mimics and IRS1 plasmid, respectively. (B) A scratch assay was performed to determine the migratory capacity of the SKOV3 cells that had been transfected with miR-126 mimics, or cotransfected with miR-126 mimics and IRS1 plasmid, respectively. (C) A Transwell assay was performed to determine the invasive capacity of the SKOV3 cells that had been transfected with miR-126 mimics, or cotransfected with miR-126 mimics and IRS1 plasmid, respectively. Magnification, x200. Control, SKOV3 cells without any transfection; ${ }^{* *} \mathrm{P}<0.01$ vs. control. IRS1, insulin receptor substrate 1 ; miR, microRNA; GAPDH, glyceraldehyde 3-phosphate dehydrogenase.

Fig. 2B, the migratory capacity of the SKOV3 cells transfected with miR-126 mimics was significantly downregulated when compared with the control group $(\mathrm{P}<0.01)$. Furthermore, it was demonstrated that miR-126 overexpression also significantly suppressed SKOV3 cell invasion $(\mathrm{P}<0.01)$. Such findings suggest that miR-126 serves inhibitory roles in the regulation of EC cell migration and invasion.

IRS1 is a target gene of miR-126. According to bioinformatical predication, IRS1 is a putative target gene of miR-126. To verify this predication, the wild-type (WT) and mutant-type (MUT) IRS1 3'-UTRs were generated (Fig. 3A). Following this, the luciferase reporter assay was performed to confirm whether miR-126 directly binds to seed sequences in the IRS1 3'-UTRs in the SKOV3 cells. As presented in Fig. 3B, the luciferase activity was significantly reduced in cells that were cotransfected with the WT IRS1 3'-UTR and miR-126 mimics $(\mathrm{P}<0.01)$; however, the luciferase activity demonstrated no difference in the SKOV3 cells that had been cotransfected with the MUT IRS1 3'-UTR and miR-126 mimics when compared with the control group, indicating that IRS1 is a target gene of miR-126 in SKOV3 cells. Subsequent to this, the SKOV3 cells were transfected with miR-126 mimics and miR-126 inhibitor, respectively, and this confirmed that the transfection efficiency was satisfactory (Fig. 3C; $<<0.01$ ). The protein level of IRS1 was then determined. As presented in Fig. 3D, the overexpression of miR-126 inhibited the protein expression of IRS1, while inhibition of miR-126 significantly enhanced the protein expression of IRS1 in the SKOV3 cells $(\mathrm{P}<0.01)$. These findings indicate that miR-126 negatively mediates the protein expression of IRS1 through its direct binding to the seed sequences in the 3'-UTR in IRS1 mRNA. 
IRS1 is involved in miR-126-mediated EC cell migration and invasion. To further investigate whether IRS1 is involved in miR-126-mediated EC cell migration and invasion, SKOV3 cells were transfected with miR-126 mimics, or were cotransfected with miR-126 mimics and IRS1 plasmid. Following transfection, the protein expression of IRS1 in each group was determined, and it was observed that transfection with IRS1 plasmid reversed the inhibitory effect of miR-126 overexpression on IRS1 protein expression (Fig. 4A; $\mathrm{P}<0.01$ ). Subsequently, the migratory and invasive capacities of the SKOV3 cells were examined in each group. As presented in Fig. 4B and C, the restoration of IRS1 reversed the suppressive effect of miR-126 overexpression on the SKOV3 cell migration and invasion $(\mathrm{P}<0.01)$. These findings indicate that IRS1 functions as a downstream effector in miR-126-mediated migration and invasion in EC SKOV3 cells.

\section{Discussion}

The migration and invasion of cancer cells are complex processes with numerous factors involved. The primary tumor cells infiltrate the adjacent tissue, and subsequently invade the systemic circulation. Here they penetrate the blood vessels and move into the distal blood capillaries, where they finally permeate into the soft tissues; this completes the migration and invasion process, thus forming a secondary tumor $(14,15)$. In the present study, the primary aim was to identify the specific role of miR-126 in the regulation of EC cell migration and invasion. It was demonstrated that miR-126, which was frequently downregulated in EC tissues, served an inhibitory role in the regulation of migration and invasion in EC SKOV3 cells. Molecular mechanism investigations established that IRS1, as a direct target of miR-126, was involved in miR-126-mediated EC cell migration and invasion. The findings suggest that miR-126/IRS1 may aid the development of therapeutics to treat EC metastasis.

In recent years, the identification of miRs has provided a novel approach for research into the development and progression of various types of human cancer (16). Furthermore, the dysregulation of miRs has been identified to be closely associated with cancer metastasis. Among the miRs involved in cancer, miR-126 has been shown to typically function as a tumor suppressor. For example, Tavazoie et al reported that the expression of miR-126 was significantly lower in breast cancer patients than that in healthy controls, and patients with an absence of miR-126 expression experienced a shorter survival period than those with miR-126 expression (17). Kim et al demonstrated that miR-126 expression was significantly downregulated in non-small cell lung cancer tissues compared with benign lung tissues, and high miR-126 expression was significantly associated with a favorable prognosis in patients with adenocarcinoma (18). However, there has not been any thorough research investigating the association between miR-126 and EC.

In the current study, it was observed that miR-126 was notably downregulated in EC tissues when compared with matched adjacent tissues. Furthermore, it was also demonstrated that miR-126 served a suppressive role in mediating EC cell migration and invasion. Notably, similar data have been reported associated with other types of cancer. Feng et al performed in vitro and in vivo experiments and observed that miR-126 had marked inhibitory effects on the migration and invasion of gastric cancer cells (19). Jia et al demonstrated that miR-126 inhibited the invasive capacity of bladder cancer cells (20). Additionally, miR-126 has also been identified to inhibit the migration and invasion of colon cancer cells (21).

The current study investigated further into the underlying mechanisms and observed that IRS1 was a direct target gene of miR-126 in the EC cells. This targeting association has also been reported in colon cancer cells and adipocytes $(22,23)$. Furthermore, it has been demonstrated that insulin-like growth factor-I receptor signaling contributes to the development of endometrial hyperplasia, the precursor to EC (24), and that IRS1 is a key mediator in oncogenic insulin-like growth factor signaling (25). Yang et al reported that IRS1 was associated with the cisplatin resistance of gastric cancer (26). IRS1 has been identified to be highly expressed in breast cancer, and regulates the sensitivity of breast cancer cells to chemotherapy (27). In the present study, it was established that upregulation of IRS1 reversed the inhibitory effect of miR-126 overexpression on EC cell migration and invasion. Shaw reported that IRS- 1 is involved in the $\alpha 4 \beta 6$ integrin-dependent activation of phosphoinositide $3-\mathrm{OH}$ kinase and the promotion of invasion (28). Additionally, IRS-1 can mediate cell migration through the formation of a dynamic complex with E-cadherin and $\alpha 5$ integrin under the control of $\alpha$-catenin (29).

Other target genes of miR-126 have also been reported in the literature. It has been noted that miR-126 inhibits the invasion of gastric cancer cells partially by targeting v-crk avian sarcoma virus CT10 oncogene homolog (Crk) (30). Liu et al reported that miR-126 inhibits the growth of gastric cancer cells by targeting phosphoinositide 3-kinase regulatory subunit 2, Crk and polo-like kinase 2 (31). Thus, the present study expands on the understanding of the miR-126 targets and the function they serve in carcinogenesis.

In conclusion, it is suggested that miR-126 may inhibit the migratory and invasive capacities of EC cells, at least partially, by inhibiting the protein expression of its target IRS1. Therefore, the continued research into the function of particular miRNAs may aid the production of molecular targeted therapy for the treatment of EC.

\section{References}

1. Banno K, Yanokura M, Iida M,Masuda K and Aoki D: Carcinogenic mechanisms of endometrial cancer: Involvement of genetics and epigenetics. J Obstet Gynaecol Res 40: 1957-1967, 2014.

2. Amant F, Moerman P, Neven P, et al: Endometrial cancer. Lancet 366: 491-505, 2005.

3. Ferlay J, Bray F, Pisani P and Parkin DM: Cancer incidence, mortality and prevalence worldwide. GLOBOCAN 2002. Cancer Incidence, Mortality and Prevalence Worldwide. IARC CancerBase 5 version 2.0. IARCPress, Lyon, France, 2004.

4. Ramón LA, Braza-Boïls A, Gilabert J, et al: microRNAs related to angiogenesis are dysregulated in endometrioid endometrial cancer. Hum Reprod 27: 3036-3045, 2012.

5. Ambros V: The functions of animal microRNAs. Nature 431: 350-355, 2004

6. Wang J, Chen X, Li P, et al: CRKL promotes cell proliferation in gastric cancer and is negatively regulated by miR-126. Chem Biol Interact 206: 230-238, 2013.

7. Huang TH and Chu TY: Repression of miR-126 and upregulation of adrenomedullin in the stromal endothelium by cancer-stromal cross talks confers angiogenesis of cervical cancer. Oncogene 33: 3636-3647, 2014 
8. Zhou Y, Feng X, Liu YL, Ye SC, Wang H, Tan WK, Tian T, Qiu YM and Luo HS: Down-regulation of miR-126 is associated with colorectal cancer cells proliferation, migration and invasion by targeting IRS-1 via the AKT and ERK1/2 signaling pathways. PLoS One 8: e81203, 2013.

9. Vergho D, Kneitz S, Rosenwald A, Scherer C, Spahn M, Burger M, Riedmiller $\mathrm{H}$ and Kneitz B: Combination of expression levels of miR-21 and miR-126 is associated with cancer-specific survival in clear-cell renal cell carcinoma. BMC Cancer 14: 25, 2014.

10. Taverna S, Amodeo V, Saieva L, Russo A, Giallombardo M, De Leo G and Alessandro R: Exosomal shuttling of miR-126 in endothelial cells modulates adhesive and migratory abilities of chronic myelogenous leukemia cells. Mol Cancer 13: 169, 2014.

11. Zhang C, Bao W, Rong Y, Yang H, Bowers K, Yeung E and Kiely M: Genetic variants and the risk of gestational diabetes mellitus: A systematic review. Hum Reprod Update 19: 376-390, 2013.

12. Copps KD and White MF: Regulation of insulin sensitivity by serine/threonine phosphorylation of insulin receptor substrate proteins IRS1 and IRS2. Diabetologia 55: 2565-2582, 2012.

13. Hua SF, Xue FX, Zhang LZ, Wang YM and Zhao J: Expression and activation of insulin receptor substrate- 1 in endometrial carcinoma. Zhonghua Fu Chan Ke Za Zhi 43: 437-441, 2008 (In Chinese).

14. Moncharmont C, Levy A, Guy JB, Falk AT, Guilbert M, Trone JC, Alphonse G, Gilormini M, Ardail D, Toillon RA, et al: Radiation-enhanced cell migration/invasion process: A review. Crit Rev Oncol Hematol 92: 133-142, 2014.

15. Wakabayashi S: A case of infantile autism who became able to communicate by writing (author's transl). Seishin Shinkeigaku Zasshi 75: 339-357, 1973 (In Japanese).

16. Bouyssou JM, Manier S, Huynh D, Issa S, Roccaro AM and Ghobrial IM: Regulation of microRNAs in cancer metastasis. Biochim Biophys Acta 1845: 255-265, 2014.

17. Tavazoie SF, Alarcón C, Oskarsson T, Padua D, Wang Q, Bos PD, Gerald WL and Massagué J: Endogenous human microRNAs that suppress breast cancer metastasis. Nature 451: 147-152, 2008.

18. Kim MK, Jung SB, Kim JS, Roh MS, Lee JH, Lee EH an Lee HW: Expression of microRNA miR-126 and miR-200c is associated with prognosis in patients with non-small cell lung cancer. Virchows Arch 465: 463-471, 2014.

19. Feng R, Chen X, Yu Y, Su L, Yu B, Li J, Cai Q, Yan M, Liu B and Zhu Z: miR-126 functions as a tumour suppressor in human gastric cancer. Cancer Lett 298: 50-63, 2010.

20. Jia AY, Castillo-Martin M, Bonal DM, Sánchez-Carbayo M, Silva JM and Cordon-Cardo C: MicroRNA-126 inhibits invasion in bladder cancer via regulation of ADAM9. Br J Cancer 110: 2945-2954, 2014.
21. Li Z, Li N, Wu M, Li X, Luo Z and Wang X: Expression of miR-126 suppresses migration and invasion of colon cancer cells by targeting CXCR4. Mol Cell Biochem 381: 233-242, 2013.

22. Li N, Li X, Huang S, Shen S and Wang X: miR-126 inhibits colon cancer proliferation and invasion through targeting IRS1, SLC7A5 and TOM1 gene. Zhong Nan Da Xue Xue Bao Yi Xue Ban 38: 809-817, 2013 (In Chinese).

23. Fernandez-Twinn DS, Alfaradhi MZ, Martin-Gronert MS, Duque-Guimaraes DE, Piekarz A, Ferland-McCollough D, Bushell $\mathrm{M}$ and Ozanne SE: Downregulation of IRS-1 in adipose tissue of offspring of obese mice is programmed cell-autonomously through post-transcriptional mechanisms. Mol Metab 3: 325-333, 2014.

24. McCampbell AS, Harris HA, Crabtree JS, Winneker RC, Walker CL and Broaddus RR: Loss of inhibitory insulin receptor substrate-1 phosphorylation is an early event in mammalian target of rapamycin-dependent endometrial hyperplasia and carcinoma. Cancer Prev Res (Phila) 3: 290-300, 2010.

25. Wang Y, Hu C, Cheng J, Chen B, Ke Q, Lv Z, Wu J and Zhou Y: MicroRNA-145 suppresses hepatocellular carcinoma by targeting IRS1 and its downstream Akt signaling. Biochem Biophys Res Commun 446: 1255-1260, 2014

26. Yang M, Shan X, Zhou X, Qiu T, Zhu W, Ding Y, Shu Y and Liu P: miR-1271 regulates cisplatin resistance of human gastric cancer cell lines by targeting IGF1R, IRS1, mTOR, and BCL2. Anticancer Agents Med Chem 14: 884-891, 2014.

27. Porter HA, Perry A, Kingsley C, Tran NL and Keegan AD: IRS1 is highly expressed in localized breast tumors and regulates the sensitivity of breast cancer cells to chemotherapy, while IRS2 is highly expressed in invasive breast tumors. Cancer Lett 338: 239-248, 2013

28. Shaw LM: Identification of insulin receptor substrate 1 (IRS-1) and IRS-2 as signaling intermediates in the alpha6beta4 integrin-dependent activation of phosphoinositide 3-OH kinase and promotion of invasion. Mol Cell Biol 21: 5082-5093, 2001.

29. Canonici A, Steelant W, Rigot V, Khomitch-Baud A, Boutaghou-Cherid H, Bruyneel E, Van Roy F, Garrouste F, Pommier G and André F: Insulin-like growth factor-I receptor, E-cadherin and alpha $v$ integrin form a dynamic complex under the control of alpha-catenin. Int J Cancer 122: 572-582, 2008

30. Li X, Wang F and Qi Y: MiR-126 inhibits the invasion of gastric cancer cell in part by targeting Crk. Eur Rev Med Pharmacol Sci 18: 2031-2037, 2014

31. Liu LY, Wang W, Zhao LY, Guo B, Yang J, Zhao XG, Hou N, Ni L, Wang AY, Song TS, et al: Mir-126 inhibits growth of SGC-7901 cells by synergistically targeting the oncogenes PI3KR 2 and Crk, and the tumor suppressor PLK2. Int J Oncol 45: 1257-1265, 2014. 